\title{
Transoral Robotic Reconstructive Surgery
}

\author{
Jesse C. Selber, MD, MPH, FACS ${ }^{1} \quad$ Karim A. Sarhane, MD, MSc ${ }^{2}$ Amir E. Ibrahim, MD ${ }^{1}$ \\ Floyd C. Holsinger, MD, FACS ${ }^{3}$
}

${ }^{1}$ Department of Plastic Surgery, University of Texas M. D. Anderson
Cancer Center, Houston, Texas
${ }^{2}$ Department of Plastic and Reconstructive Surgery, Johns Hopkins
School of Medicine, Baltimore, Maryland
${ }^{3}$ Department of Otolaryngology-Head \& Neck Surgery, Stanford
University School of Medicine, Stanford, California

Address for correspondence Jesse C. Selber, MD, MPH, FACS, Department of Plastic Surgery, MD Anderson Cancer Network, 1400 Pressler Street, Houston, TX 77030 (e-mail: jcselber@mdanderson.org).

Semin Plast Surg 2014;28:35-38.

\begin{abstract}
Keywords

- robotics

- head and neck neoplasms

- head and neck reconstruction

- free flaps

Transoral robotic surgery (TORS) has emerged as a technique that allows head and neck surgeons to safely resect large and complex oropharyngeal tumors without dividing the mandible or performing a lip-split incision. These resections provide a reconstructive challenge because the cylinder of the oropharynx remains closed and both physical access and visualization of oropharyngeal anatomy is severely restricted. Transoral robotic reconstruction (TORRS) of such defects allows the reconstructive surgeon to inset free flaps or perform adjacent tissue transfer while seeing what the resecting surgeon sees. Early experience with this technique has proved feasible and effective. Robotic reconstruction has many distinct advantages over conventional surgery, and offers patients a less morbid surgical course. In this review, we discuss the clinical applicability of transoral robotic surgery in head and neck reconstruction, highlighting the benefits and limitations of such an approach, and outlining the guidelines for its utilization.
\end{abstract}

Over the past decade, robotic surgery has opened new horizons in minimally invasive procedures. Robotic applications have officially entered the field of plastic surgery, capitalizing on two principle advantages: improved visualization and improved precision. One area where both of these features provide benefit is in reconstruction following minimally invasive extirpation of oropharyngeal tumors such as in transoral robotic surgery (TORS). In such cases, plastic surgeons face a considerable reconstructive challenge as they attempt to contour and inset vascularized tissue in a highly anatomically restricted field, limiting both dexterity and visualization. Transoral robotic reconstructive surgery (TORRS), ${ }^{1}$ whether using free flaps, local flaps, or primary closure, appears to be a superior approach in select cases, and holds the promise of expanding indications for minimally invasive reconstructive procedures. In this review, we discuss the history, feasibility, and clinical considerations of such an approach for oropharyn- geal reconstruction, and outline the guidelines for its indication.

\section{Evolution of Early-Stage Oropharyngeal Tumor Extirpation: From Open Surgery to Chemoradiation Therapy to TORS}

Traditionally, resection of oropharyngeal tumors entailed division of the mandible and sometimes the lip, or access through a large pharyngotomy, wide enough to both resect and extract the entire tumor (-Fig. 1). However, such procedures often resulted in considerable morbidity and esthetic and functional compromise. Alternatively, chemoradiation has proven to be effective in the management of some of these early-stage tumors, and has led to a paradigm shift away from extensive resections. ${ }^{2,3}$ Definitive chemoradiation, although intending to preserve anatomy, often significantly impairs laryngeal function including speech and swallowing
Issue Theme Robotics in Plastic Surgery; Guest Editor, Jesse C. Selber, MD, MPH, FACS
Copyright (C) 2014 by Thieme Medical Publishers, Inc., 333 Seventh Avenue, New York, NY 10001, USA. Tel: +1(212) 584-4662.
DOI http://dx.doi.org/ 10.1055/s-0034-1368166. ISSN 1535-2188. 


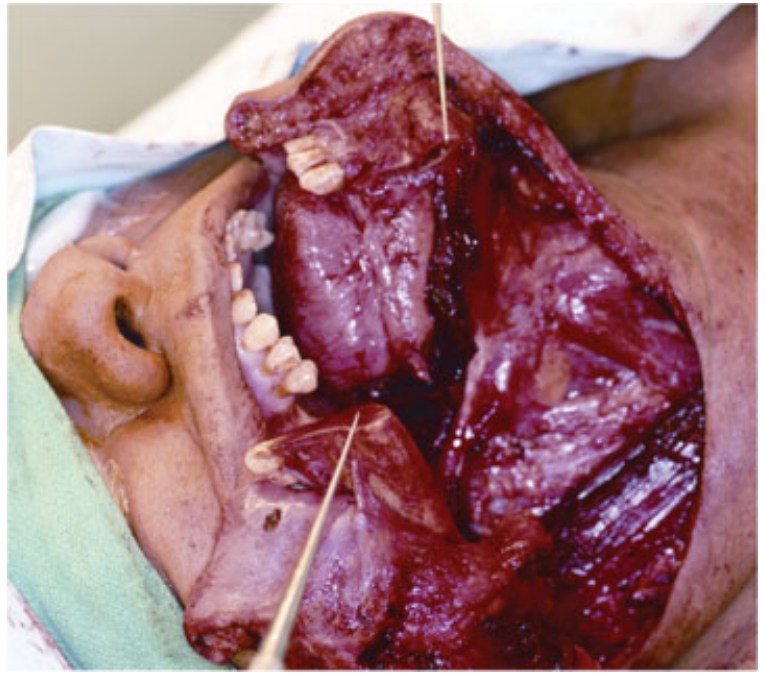

Fig. 1 Traditional approaches to early-stage tumors of the oropharynx require division of the lip and mandible and release of the tongue base, resulting in significant aesthetic and functional morbidity.

due to the local fibrosing effects of radiation. Studies evaluating intermediate and long-term adverse events following chemotherapy and irradiation showed that patients may still suffer from significant functional impairment, including persistent swallowing difficulties and dysphagia, xerostomia, diminished vocal ability, and airway compromise with a higher risk for aspiration. ${ }^{4-7}$ In one study, chemoradiation therapy was shown to be associated with $4 \%$ treatmentrelated death, $6 \%$ osteoradionecrosis, $12 \%$ chronic aspiration, $12 \%$ nonhealing ulcers, and $42 \%$ serious adverse events. ${ }^{8}$ For these reasons, the pendulum has swung back in the direction of a more reasonable middle ground between debilitating surgery and debilitating chemoradiation.

Transoral robotic surgery (TORS) emerged as a prominent surgical approach for the successful resection of oropharyngeal tumors without division of the lip and mandible. This technique can offer the benefits of locoregional tumor control without the complications associated with open access or high-dose chemoradiation. Minimally invasive resections are achieved either by TORS alone or "pull-through" procedures where TORS is used to approach the tumor transorally and a small lateral pharyngotomy is used to complete the extirpation and deliver the tumor. TORS was adopted by multiple groups, and obtained approval from the Food and Drug Administration in $2009 .{ }^{9}$ Midterm results have been promising; in one study evaluating functional outcomes, only $9 \%$ of patients required temporary tracheostomy tubes, all of whom were decannulated by postoperative day $14 .^{10}$ In another study prospectively assessing functional outcomes, 31\% required tracheostomy, but all were decannulated after $\sim 7$ days after surgery. ${ }^{11}$ Evaluation of long-term functional and oncologic outcomes is ongoing, but these preliminary results suggest that minimally invasive approaches may have good functional outcomes and mitigate some of the disadvantages of alternative techniques. Traditionally, TORS resections are left to granulate, limiting the scope of surgical resections to wounds that can safely heal by secondary intention. Ultimately, to expand the usefulness of TORS, reconstruction of certain select defects is required.

\section{Emergence and Feasibility of Transoral Robotic Reconstructive Surgery}

Minimally invasive resections, including both TORS and pullthroughs, create a considerable reconstructive challenge for the plastic surgeon. Although the current practice following TORS resections is to allow healing by secondary intention, many defects are large and complex enough to benefit softtissue coverage. In addition, when the carotid sheath is exposed, an oro- or pharyngocutaneous fistula is created, or velopharyngeal compromise is anticipated, some form of vascularized tissue is required for anatomical restoration and appropriate long-term function. In such cases, accurate suture placement for flap insetting is limited by a confined working space and suboptimal vision. Specifically, approaching the anatomical region bound by the uvula and the epiglottis is of particular challenge without wide exposure. Preserving a competent velopharyngeal sphincter, a watertight seal between the pharynx and neck, and adequate sensation and volume in the tongue base are necessary to optimize the physiological function of the oropharynx and minimize functional deficits. ${ }^{12,13}$ For these cases, transoral inset of a free flap or local flap using robotic surgical visualization and precision would be an appealing reconstructive strategy. The technique requires a mouth retractor to set the interdental opening, and two robotic arms and an endoscope converging on the target oropharyngeal anatomy (-Fig. 2). This technique has proven to be both feasible ${ }^{14}$ and effective. ${ }^{15}$ In addition, because TORRS allows reconstruction of more extensive defects in deep portions of the oropharynx, head and neck surgeons are able to enlarge the boundaries of what is considered resectable using TORS.

\section{Indications for TORRS}

Given the relative infancy of this procedure, clear guidelines on the use of TORRS are still lacking. Existing literature, so far, has focused on feasibility, safety, and application of the robot in reconstruction. Recently, we introduced, through a small series of 20 patients undergoing TORS, a conceptual framework based on tumor location, tumor extent, prior treatment, and patient-specific criteria. ${ }^{16}$

\section{Tumor Site}

The location of the primary tumor is probably the most significant determinant of both the feasibility of TORS resection and reconstruction.

Tumors within the oral cavity do not routinely need TORS as they often have good direct access. The exception is the retromolar trigone; large tumors in this area are in proximity to the base of tongue (BOT), tonsil, and mandibular ramus and may thus benefit from TORS resection and reconstruction. Local flaps such as the facial artery myomucosal flap, buccal fat pad flap, and buccal and pharyngeal mucosal 

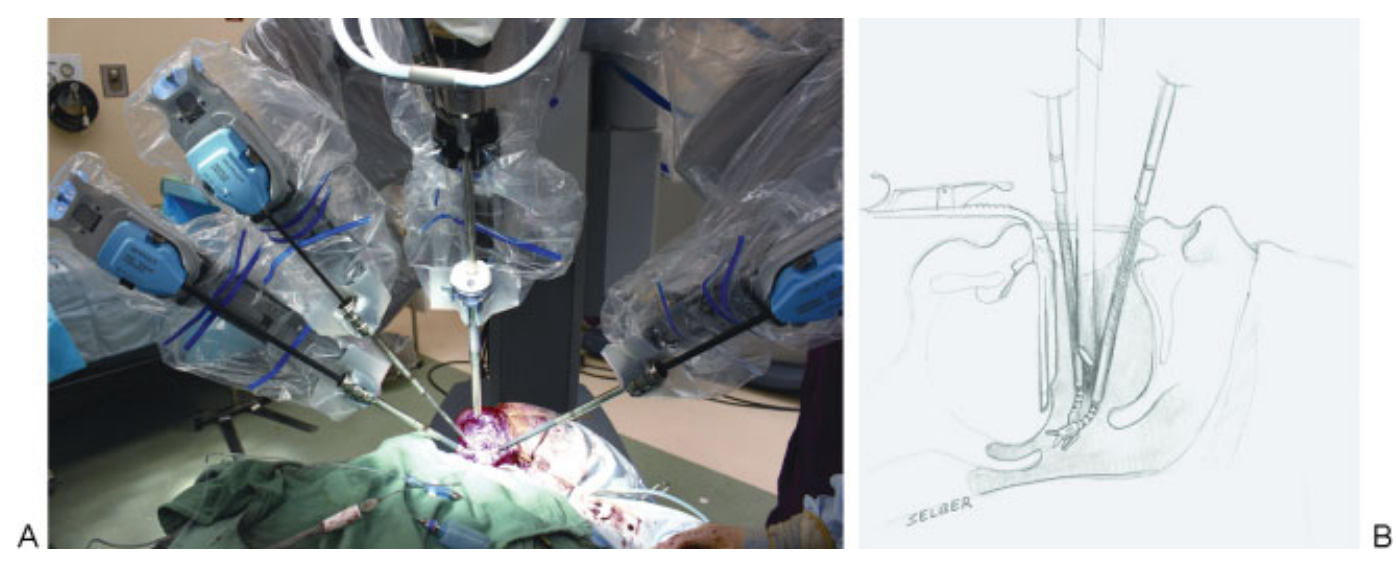

Fig. 2 Transoral robotic reconstruction requires a mouth retractor to set the interdental opening. The robotic endoscope and two robotic instrument arms are introduced through the mouth and converge on the target oropharyngeal anatomy. External view (A) and depiction of internal view (B) is shown.

transposition flaps are sufficient for reconstructing many such cases. Rarely is a free flap needed in this area, which is quite compact; in addition, a free flap would necessitate exposing the neck to the oropharynx, so unless there are clear indications (such as prior radiation or resections), a free flap can often be avoided.

Tumors within the oropharynx (tonsil, BOT, soft palate) on the other hand constitute the most common indication for TORS. Although transoral resection of such tumors by other techniques has long been performed, ${ }^{17,18}$ the robot affords improved optical fields and more precise instrumentation, making resection of more complex and invasive tumors possible. Such large lesions in addition to those exposing critical structures (carotid sheath or bone) may benefit from robotic free-flap reconstruction. As for tumors of the soft palate, free flaps or palatoplasties can be useful, but it is important to remember that equivalent functional outcomes may be achieved with a prosthetic obturator in certain situations. ${ }^{19,20}$

For supraglottic laryngeal tumors, reconstruction is determined by whether the lesion is above or below the hyoid and the amount of hypopharyngeal disease present. In patients with a large oral opening, the supra- and infrahyoid areas can be easily accessed for TORS resection and reconstruction might not be required, depending on the case. Moreover, free-tissue transfer is not always feasible in these regions. However, in case the defect is large and would require a free flap, these space-occupying flaps will likely require a tracheotomy. Risk of airway compromise is increased in this area even if partial dehiscence of a flap occurs, so conservative management of the trach is advised.

\section{Tumor Extent}

The current approach following robotic-assisted resection of $\mathrm{T} 1$ and $\mathrm{T} 2$ tumors is healing by secondary intention. This practice is feasible for smaller tumors and results in good functional outcomes; however, for larger lesions (T3, T4, or even posterior T2 tumors) or when the carotid sheath is exposed, surgical fistula is created, or velopharyngeal compromise is anticipated to occur, vascularized soft tissue reconstruction is a better option for anatomical restoration. Some tumors may undergo "hybrid" resections where a transoral approach is coupled with a small pharyngotomy; in such cases, a "hybrid" reconstruction is indicated, where TORRS is performed and the deepest portion of the inset is completed through the neck.

\section{Prior Therapy}

For patients with a history of neck irradiation, free-flap reconstruction is often beneficial. The rationale is that bringing in healthy vascularized tissue can have a nourishing effect on surrounding tissue, and improve the long-term stability of the repair. Free-tissue reconstruction may also permit reirradiation, a frequently encountered scenario. Because of radiation-induced vascular injury, local flaps are not recommended in such settings, even for smaller defects.

\section{Patient Factors}

General performance status ultimately determines the decision for any type of surgery. As the obesity epidemic is growing at a very rapid rate, ${ }^{21}$ medical morbidities such as diabetes and atherosclerosis are becoming more and more common among patients. Such conditions negatively impact perfusion, wound healing and hence increase the risk of dehiscence, infections, and other wound complications. Proper glycemic control in the pre- and postoperative period is strongly recommended. Despite the high rate of obesity, however, head and neck patients are more often cachectic, malnourished, and poorly maintained. Significant smoking history and poor cardiopulmonary status may render certain patients unable to tolerate long operative times under anesthesia. It is also important to consider other preoperative patient variables that are notoriously associated with postoperative morbidity, such as anemia, ${ }^{22,23}$ coronary or peripheral vascular disease, dysphagia, or a history of recurrent aspirations.

As enthusiasm continues to mount for TORS, and reconstructive surgeons must keep pace with the growing demands for reconstruction in these settings, it is critical to have a rational approach. We feel these considerations help 
guide our approach to TORRS and will serve as a primer for others adopting TORRS in their practice.

\section{Limitations of TORRS}

Certain specific financial, logistic, and technical limitations of TORRS should be considered. There is an expense to robotic surgery of any kind, although when being used in combination with robotic head and neck procedures, the additional cost is mitigated. Logistically, bringing in and setting up the surgical robot takes additional time, staff, and experience. This can make an already long case longer, and requires extensive staff training and repetition to make efficient. Technically, the set-up is very sensitive to small changes. Movements at the tips of the instruments correspond to larger movements proximally where excursion of the arms is limited by the area within the mouth retractor. Set-up will often require readjustment; care must be taken to protect the teeth and more distantly the eyes from iatrogenic injury. Finally, because of our strict criteria for use of TORRS, the number of cases for which this technology is indicated is relatively small unless the head and neck practice is very high volume.

\section{Conclusion}

Robotic-assisted head and neck cancer surgery is an alternative approach for the management of oropharyngeal tumors, but necessitates the development of appropriate reconstructive methods. TORRS represents a bourgeoning robotic transoral reconstructive technique and may eventually be a critical part of any robust TORS program. A rational approach to the use of the robot in transoral reconstruction will help guide the development of this field.

\section{References}

1 Selber J. Discussion: robotic-assisted FAMM flap for soft palate reconstruction. Laryngoscope 2014 Jan 28; [Epub ahead of print]

2 The Department of Veterans Affairs Laryngeal Cancer Study Group. Induction chemotherapy plus radiation compared with surgery plus radiation in patients with advanced laryngeal cancer. $\mathrm{N}$ Engl J Med 1991;324(24):1685-1690

3 Ozer E, Waltonen J. Transoral robotic nasopharyngectomy: a novel approach for nasopharyngeal lesions. Laryngoscope $2008 ; 118(9)$ : 1613-1616

4 Campbell BH, Spinelli K, Marbella AM, Myers KB, Kuhn JC, Layde PM. Aspiration, weight loss, and quality of life in head and neck cancer survivors. Arch Otolaryngol Head Neck Surg 2004;130(9): $1100-1103$

5 Kotz T, Costello R, Li Y, Posner MR. Swallowing dysfunction after chemoradiation for advanced squamous cell carcinoma of the head and neck. Head Neck 2004;26(4):365-372

6 Rademaker AW, Vonesh EF, Logemann JA, et al. Eating ability in head and neck cancer patients after treatment with chemoradia- tion: a 12-month follow-up study accounting for dropout. Head Neck 2003;25(12):1034-1041

7 Roh J-L, Kim A-Y, Cho MJ. Xerostomia following radiotherapy of the head and neck affects vocal function. J Clin Oncol 2005;23(13): 3016-3023

8 Machtay M, Rosenthal DI, Hershock D, et al; Penn Cancer Center Clinical Trials Group. Organ preservation therapy using induction plus concurrent chemoradiation for advanced resectable oropharyngeal carcinoma: a University of Pennsylvania Phase II Trial. J Clin Oncol 2002;20(19):3964-3971

9 Ibrahim AE, Sarhane KA, Baroud JS, Atiyeh BS. Robotics in plastic surgery, a review. Eur J Plast Surg 2012;35(8):571-578

10 Iseli TA, Kulbersh BD, Iseli CE, Carroll WR, Rosenthal EL, Magnuson JS. Functional outcomes after transoral robotic surgery for head and neck cancer. Otolaryngol Head Neck Surg 2009;141(2):166-171

11 Moore EJ, Olsen KD, Kasperbauer JL. Transoral robotic surgery for oropharyngeal squamous cell carcinoma: a prospective study of feasibility and functional outcomes. Laryngoscope 2009;119(11): 2156-2164

12 Song HG, Yun IS, Lee WJ, Lew DH, Rah DK. Robot-assisted free flap in head and neck reconstruction. Arch Plast Surg 2013;40(4): 353-358

13 de Almeida JR, Park RCW, Genden EM. Reconstruction of transoral robotic surgery defects: principles and techniques. J Reconstr Microsurg 2012;28(7):465-472

14 Selber JC, Robb G, Serletti JM, Weinstein G, Weber R, Holsinger FC. Transoral robotic free flap reconstruction of oropharyngeal defects: a preclinical investigation. Plast Reconstr Surg 2010;125(3): 896-900

15 Selber JC. Transoral robotic reconstruction of oropharyngeal defects: a case series. Plast Reconstr Surg 2010;126(6):1978-1987

16 Longfield EA, Holsinger FC, Selber JC. Reconstruction after robotic head and neck surgery: when and why. J Reconstr Microsurg 2012; 28(7):445-450

17 Holsinger FC, McWhorter AJ, Ménard M, Garcia D, Laccourreye O. Transoral lateral oropharyngectomy for squamous cell carcinoma of the tonsillar region: I. Technique, complications, and functional results. Arch Otolaryngol Head Neck Surg 2005;131(7):583-591

18 Laccourreye O, Hans S, Ménard M, Garcia D, Brasnu D, Holsinger FC. Transoral lateral oropharyngectomy for squamous cell carcinoma of the tonsillar region: II. An analysis of the incidence, related variables, and consequences of local recurrence. Arch Otolaryngol Head Neck Surg 2005;131(7):592-599

19 Rieger J, Bohle Iii G, Huryn J, Tang JL, Harris J, Seikaly H. Surgical reconstruction versus prosthetic obturation of extensive soft palate defects: a comparison of speech outcomes. Int J Prosthodont 2009;22(6):566-572

20 Marsh JL, Wray RC. Speech prosthesis versus pharyngeal flap: a randomized evaluation of the management of velopharyngeal incompetency. Plast Reconstr Surg 1980;65(5):592-594

21 Atiyeh BS, Sarhane KA. Overweight and obesity: a true global epidemic. In: Atiyeh BS, Costagliola M, eds. Body Contouring Following Bariatric Surgery and Massive Weight Loss Post-Bariatric Body Contouring. Sharjah, United Arab Emirates: Bentham Science Publishers; 2012:3-11

22 Sarhane KA, Flores JM, Cooney CM, et al. Preoperative anemia and postoperative outcomes in immediate breast reconstructive surgery: a critical analysis of 10,958 patients from the ACS-NSQIP database. Plast Reconstr Surg 2013; in press

23 Hill JB, Patel A, Del Corral GA, et al. Preoperative anemia predicts thrombosis and free flap failure in microvascular reconstruction. Ann Plast Surg 2012;69(4):364-367 